\title{
A FINITE-DIMENSIONAL REDUCTION METHOD FOR SLIGHTLY SUPERCRITICAL ELLIPTIC PROBLEMS
}

\author{
RICCARDO MOLLE AND DONATO PASSASEO
}

Received 27 August 2003

We describe a finite-dimensional reduction method to find solutions for a class of slightly supercritical elliptic problems. A suitable truncation argument allows us to work in the usual Sobolev space even in the presence of supercritical nonlinearities: we modify the supercritical term in such a way to have subcritical approximating problems; for these problems, the finite-dimensional reduction can be obtained applying the methods already developed in the subcritical case; finally, we show that, if the truncation is realized at a sufficiently large level, then the solutions of the approximating problems, given by these methods, also solve the supercritical problems when the parameter is small enough.

\section{Introduction}

Finite-dimensional reduction methods have been used in several contexts to find solutions of differential equations. In problems where some concentration phenomena arise, these methods are used to construct blowing-up solutions and to describe the effect of the domain shape on the existence and on the number of solutions of this type.

This situation occurs, for example, in some nonlinear elliptic problems involving critical (or nearly critical) Sobolev exponents. In this case, these methods are used to point out the role of Green's and Robin's functions to construct multispike solutions, and to describe the lack of compactness and the concentration phenomena related to the presence of critical or nearly critical nonlinearities.

Several works have been devoted to the case of critical or subcritical nonlinear problems (see, e.g., $[1,2,3,5,7,11,12,13,14,15])$.

More recently, the case of slightly supercritical nonlinearities has been also considered (see $[6,8,9,10]$ and the references therein). It is clear that for these problems, an additional difficulty is due to the fact that the Sobolev embedding is no longer valid.

In [6], the authors consider directly the supercritical problem. So, in order to carry out the finite-dimensional reduction, they need some uniform estimates in suitably chosen spaces.

In the present paper, we describe a different approach: by a modification of the nonlinear term at infinity, we approximate our problems by subcritical (indeed, asymptotically 


\section{Finite-dimensional reduction for supercritical problems}

linear) problems. Then, we obtain the finite-dimensional reduction for the approximating problems, applying directly the methods already developed for the critical and subcritical cases (see, e.g., $[1,3,13,14,15])$. Finally, we show that the solutions of the approximating problems, given by these methods, also solve the supercritical problems when the parameter is small enough. In this way, we can work in the usual Sobolev space; we only need estimates in $H_{0}^{1}$ (indeed, one can also obtain, as a by-product, more careful uniform estimates, but they are not required to carry out the finite-dimensional reduction).

A similar approach is used in [16] to find solutions for problems of this type in unbounded domains (see Remark 2.6).

\section{Approximating problems and finite-dimensional reduction}

Our aim is to find solutions of the following problem:

$$
\begin{gathered}
-\Delta u=u^{(n+2) /(n-2)+\varepsilon} \quad \text { in } \Omega, \\
u>0 \quad \text { in } \Omega, \\
u=0 \quad \text { on } \partial \Omega,
\end{gathered}
$$

where $\Omega$ is a bounded domain of $\mathbb{R}^{n}, n \geq 3$, and $\varepsilon$ is a small positive parameter.

We consider the functions

$$
\bar{U}_{\xi, \mu}(x)=[n(n-2)]^{(n-2) / 4}\left(\frac{\mu}{\mu^{2}+|x-\xi|^{2}}\right)^{(n-2) / 2} \quad \forall \xi \in \mathbb{R}^{n}, \mu>0 .
$$

Denote by $U_{\xi, \mu}$ the projection of $\bar{U}_{\xi, \mu}$ onto $H_{0}^{1}(\Omega)$, namely, the solution of the problem

$$
\begin{gathered}
-\Delta U_{\xi, \mu}=\bar{U}_{\xi, \mu}^{(n+2) /(n-2)} \text { in } \Omega, \\
U_{\xi, \mu}=0 \quad \text { on } \partial \Omega .
\end{gathered}
$$

For $k \in \mathbb{N}$ and $\varepsilon$ small enough, we look for solutions of (2.1) of the form

$$
u_{k, \mathcal{\varepsilon}}(\xi, \lambda)=\sum_{i=1}^{k} U_{i, \mathcal{\varepsilon}}(\xi, \lambda)+\theta_{k, \mathcal{\varepsilon}}(\xi, \lambda)
$$

where $\xi=\left(\xi_{1}, \ldots, \xi_{k}\right) \in \Omega^{k}, \lambda=\left(\lambda_{1}, \ldots, \lambda_{k}\right) \in\left(\mathbb{R}^{+}\right)^{k}, U_{i, \mathcal{\varepsilon}}(\xi, \lambda)=U_{\xi_{i},\left(\lambda_{i}^{2} \varepsilon\right)^{1 /(n-2)}}$, and $\theta_{k, \varepsilon}(\xi, \lambda)$ $\rightarrow 0$ as $\varepsilon \rightarrow 0$.

For $\delta \in] 0,1[$ small enough, consider the set

$$
\begin{aligned}
& M_{\delta}=\left\{(\xi, \lambda) \in \Omega^{k} \times\left(\mathbb{R}^{+}\right)^{k}: \operatorname{dist}\left(\xi_{i}, \partial \Omega\right)>\delta,\right. \\
&\left.\delta<\lambda_{i}<\frac{1}{\delta},\left|\xi_{i}-\xi_{j}\right|>\delta \text { if } i \neq j(i, j=1, \ldots, k)\right\} .
\end{aligned}
$$

We fix a constant $c$ such that

$$
c>[n(n-2)]^{(n-2) / 4} \frac{1}{\delta}
$$


(notice that $[n(n-2)]^{(n-2) / 4}(1 / \delta)=\max \bar{U}_{\xi, \delta^{2 /(n-2)}}$ for all $\left.\xi \in \mathbb{R}^{n}\right)$; then set $t_{\varepsilon}=c / \sqrt{\varepsilon}$ and define $g_{\varepsilon}: \mathbb{R} \rightarrow \mathbb{R}^{+}$as follows:

$$
g_{\varepsilon}(t)= \begin{cases}0 & \text { if } t \leq 0 \\ t^{(n+2) /(n-2)+\varepsilon} & \text { if } 0 \leq t \leq t_{\varepsilon} \\ t_{\varepsilon}^{(n+2) /(n-2)+\varepsilon}+\left(\frac{n+2}{n-2}+\varepsilon\right) t_{\varepsilon}^{4 /(n-2)+\varepsilon}\left(t-t_{\varepsilon}\right) & \text { if } t \geq t_{\varepsilon}\end{cases}
$$

Let $J_{\varepsilon}: H_{0}^{1}(\Omega) \rightarrow \mathbb{R}$ be the functional defined by

$$
J_{\varepsilon}(u)=\frac{1}{2} \int_{\Omega}|\nabla u|^{2} d x-\int_{\Omega} G_{\varepsilon}(u) d x
$$

where $G_{\varepsilon}(t)=\int_{0}^{t} g_{\varepsilon}(\tau) d \tau$.

Remark 2.1. Notice that $G_{\varepsilon}(t)$ has subcritical growth at infinity for all $\varepsilon>0$; so $J_{\varepsilon}$ is a well-defined $C^{2}$ functional in $H_{0}^{1}(\Omega)$. Therefore, we can work in the usual Sobolev spaces and apply the finite-dimensional reduction methods already developed for critical or subcritical problems; in particular, taking also into account the choice of the constant $c$, we can obtain the following two lemmas (indeed, these methods have been first applied to problems with homogeneous nonlinearities, but all the arguments still work if the homogeneous nonlinear terms are replaced by $\left.g_{\varepsilon}(u)\right)$.

Lemma 2.2. There exists $\varepsilon_{k}>0$ such that, for each $\left.\varepsilon \in\right] 0, \varepsilon_{k}[$, there exists a smooth map $\theta_{k, \varepsilon}: M_{\delta} \rightarrow H_{0}^{1}(\Omega)$ satisfying the following property: the function $u_{k, \varepsilon}(\xi, \lambda)($ see (2.4)), with $(\xi, \lambda) \in M_{\delta}$, is a critical point for $J_{\varepsilon}$ if and only if $(\xi, \lambda)$ is a critical point for the function

$$
F_{k, \varepsilon}(\xi, \lambda)=J_{k, \varepsilon}\left(u_{k, \varepsilon}(\xi, \lambda)\right) .
$$

Moreover,

$$
\lim _{\varepsilon \rightarrow 0} \sup \left\{\left\|\theta_{\varepsilon}(\xi, \lambda)\right\|_{H_{0}^{1}(\Omega)}:(\xi, \lambda) \in M_{\delta}\right\}=0 .
$$

The proof is similar to the proof of [16, Proposition 3.2]. An asymptotic expansion of the finite-dimensional reduced functional $F_{k, \varepsilon}$ is given in the following lemma which can be proved arguing as in $[3,15]$ (with $\varepsilon$ replaced by $-\varepsilon$ ).

Lemma 2.3. Let $F_{k, \varepsilon}: M_{\delta} \rightarrow \mathbb{R}$ be the function defined by (2.9). Then

$$
F_{k, \varepsilon}(\xi, \lambda)=k S_{n}+k \bar{a}_{n} \varepsilon \lg \varepsilon+\varepsilon\left[k \bar{b}_{n}+\bar{c}_{n} \psi_{k}(\xi, \lambda)\right]+\varphi_{k, \varepsilon}(\xi, \lambda),
$$

where $S_{n}, \bar{a}_{n}, \bar{b}_{n}$, and $\bar{c}_{n}$ are suitable constants depending only on $n, \varepsilon^{-1} \varphi_{k, \varepsilon} \rightarrow 0$ in $C^{1}\left(M_{\delta}\right)$ as $\varepsilon \rightarrow 0$, and

$$
\psi_{k}(\xi, \lambda)=\frac{\bar{p}_{n}}{2}\left[\sum_{i=1}^{k} H\left(\xi_{i}, \xi_{i}\right) \lambda_{i}^{2}-2 \sum_{1 \leq i<j \leq k} G\left(\xi_{i}, \xi_{j}\right) \lambda_{i} \lambda_{j}\right]+\sum_{i=1}^{k} \lg \lambda_{i},
$$

where $\bar{p}_{n}$ is a positive constant depending only on $n, G(x, y)$ denotes the Green function of $-\Delta$ with zero Dirichlet condition on the boundary of $\Omega$, and $H(x, y)$ is its regular part. 
686 Finite-dimensional reduction for supercritical problems

Remark 2.4. As a consequence of Lemmas 2.2 and 2.3, every critical point for $\psi_{k}$, which persists with respect to small $C^{1}$ perturbations, for $\varepsilon$ small enough, gives rise to a solution of the form (2.4) for the equation $-\Delta u=g_{\varepsilon}(u)$. Indeed, for $\varepsilon$ small enough, this solution also solves problem (2.1), as we can infer from the following proposition.

Proposition 2.5. Assume that there exists $\bar{\varepsilon}_{k}>0$ such that, for all $\left.\varepsilon \in\right] 0, \bar{\varepsilon}_{k}[$, the function $F_{k, \varepsilon}$ has a critical point $\left(\xi_{\varepsilon}, \lambda_{\varepsilon}\right) \in M_{\delta}$. Then there exists $\varepsilon_{k}^{\prime}>0$ such that

$$
\left.\sup _{\Omega} u_{k, \varepsilon}\left(\xi_{\varepsilon}, \lambda_{\varepsilon}\right)<t_{\varepsilon} \quad \forall \varepsilon \in\right] 0, \bar{\varepsilon}_{k}^{\prime}[
$$

( $t_{\varepsilon}$ appeared in the definition of $\left.g_{\varepsilon}\right)$.

Proof. For all $\varepsilon>0$, set $\Omega_{\varepsilon}=\varepsilon^{-1 /(n-2)} \Omega$ and

$$
v_{\varepsilon}(y)=\varepsilon^{2 /(4+(n-2) \varepsilon)} u_{k, \varepsilon}\left(\xi_{\varepsilon}, \lambda_{\varepsilon}\right)\left(\varepsilon^{1 /(n-2)} y\right) \quad \forall y \in \Omega_{\varepsilon} .
$$

Clearly, our assertion is equivalent to proving that, for $\varepsilon$ small enough, $\sup _{\Omega_{\varepsilon}} v_{\varepsilon}<\tau_{\varepsilon}$, where $\tau_{\varepsilon}=t_{\varepsilon} \varepsilon^{2 /(4+(n-2) \varepsilon)}$.

Notice that $v_{\varepsilon} \in H_{0}^{1}\left(\Omega_{\varepsilon}\right)$ and solves the problem

$$
\begin{aligned}
-\Delta v_{\varepsilon} & =\tilde{g}_{\varepsilon}\left(v_{\varepsilon}\right) \quad \text { in } \Omega_{\varepsilon}, \\
v_{\varepsilon} & =0 \quad \text { on } \partial \Omega_{\varepsilon},
\end{aligned}
$$

where

$$
\tilde{g}_{\varepsilon}(\tau)= \begin{cases}0 & \text { if } \tau \leq 0 \\ \tau^{(n+2) /(n-2)+\varepsilon} & \text { if } 0 \leq \tau \leq \tau_{\varepsilon} \\ \tau_{\varepsilon}^{(n+2) /(n-2)+\varepsilon}+\left(\frac{n+2}{n-2}+\varepsilon\right) \tau_{\varepsilon}^{4 /(n-2)+\varepsilon}\left(\tau-\tau_{\varepsilon}\right) & \text { if } \tau \geq \tau_{\varepsilon}\end{cases}
$$

Moreover, we have

$$
\lim _{\varepsilon \rightarrow 0} \tau_{\varepsilon}=c
$$

Because of the choice of $c$ (see (2.6)), we can choose another constant $c_{1}$ such that

$$
\frac{1}{\delta}[n(n-2)]^{(n-2) / 4}<c_{1}<c .
$$

Since $\theta_{k, \varepsilon}\left(\xi_{\varepsilon}, \lambda_{\varepsilon}\right) \rightarrow 0$, as $\varepsilon \rightarrow 0$, in $H_{0}^{1}(\Omega)$ (hence also in $L^{2 n /(n-2)}(\Omega)$ ) and since $c_{1}>$ $(1 / \delta)[n(n-2)]^{(n-2) / 4}$, from the definition of $v_{\varepsilon}$, it follows that $\lim _{\varepsilon \rightarrow 0}$ meas $\left(A_{\varepsilon}\right)=0$, where $A_{\varepsilon}=\left\{x \in \Omega_{\varepsilon}: v_{\varepsilon}(x)>c_{1}\right\}$.

If $A_{\varepsilon}=\varnothing$ for $\varepsilon$ small enough, then the assertion is proved (because $\lim _{\varepsilon \rightarrow 0} \tau_{\varepsilon}=c>c_{1}$ ).

On the contrary, if meas $\left(A_{\varepsilon}\right)>0$ for $\varepsilon$ small (up to a subsequence), set $\hat{v}_{\varepsilon}=v_{\varepsilon}-c_{1}$ and notice that $\hat{v}_{\varepsilon}$ satisfies

$$
\begin{gathered}
-\Delta \hat{v}_{\varepsilon}=\tilde{g}_{\varepsilon}\left(c_{1}+\hat{v}_{\varepsilon}\right) \quad \text { in } A_{\varepsilon}, \\
\hat{v}_{\varepsilon}=0 \quad \text { on } \partial A_{\varepsilon} .
\end{gathered}
$$


Now, observe that, for $\varepsilon$ small enough (so that $c_{1} \leq \tau_{\varepsilon}$ ), we have

$$
\tilde{g}_{\varepsilon}\left(c_{1}+\hat{\tau}\right) \leq c_{1}^{(n+2) /(n-2)+\varepsilon}+\left(\frac{n+2}{n-2}+\varepsilon\right) \tau_{\varepsilon}^{4 /(n-2)+\varepsilon} \hat{\tau} \quad \forall \hat{\tau} \geq 0
$$

which implies $\sup _{A_{\varepsilon}} \hat{v}_{\varepsilon} \leq \sup _{B_{\varepsilon}} \tilde{v}_{\varepsilon}$, where $B_{\varepsilon}$ is a ball in $\mathbb{R}^{n}$ such that meas $\left(B_{\varepsilon}\right)=\operatorname{meas}\left(A_{\varepsilon}\right)$ and $\tilde{v}_{\varepsilon}$ is the solution of the problem

$$
\begin{gathered}
-\Delta \tilde{v}_{\varepsilon}=c_{1}^{(n+2) /(n-2)+\varepsilon}+\left(\frac{n+2}{n-2}+\varepsilon\right) \tau_{\varepsilon}^{4 /(n-2)+\varepsilon} \tilde{v}_{\varepsilon} \quad \text { in } B_{\varepsilon} \\
\tilde{v}_{\varepsilon}=0 \quad \text { on } \partial B_{\varepsilon} .
\end{gathered}
$$

Notice that, since meas $\left(B_{\varepsilon}\right) \rightarrow 0$ while $c_{1}^{(n+2) /(n-2)+\varepsilon}$ and $((n+2) /(n-2)+\varepsilon) \tau_{\varepsilon}^{4 /(n-2)+\varepsilon}$ remain bounded as $\varepsilon \rightarrow 0$, problem (2.21) has indeed a unique solution $\tilde{v}_{\varepsilon}$ for $\varepsilon$ small enough and, moreover, $\lim _{\varepsilon \rightarrow 0} \sup _{B_{\varepsilon}} \tilde{v}_{\varepsilon}=0$.

Therefore, since $\tau_{\varepsilon} \rightarrow c$ as $\varepsilon \rightarrow 0$ and $c>c_{1}$, for $\varepsilon$ small enough, we have $\sup _{\Omega_{\varepsilon}} v_{\varepsilon}<\tau_{\varepsilon}$, which is our claim.

Remark 2.6. In [16], Yan uses a similar method to find solutions of problems of the form

$$
\begin{gathered}
-\Delta u=Q(y) u^{(n+2) /(n-2)+\varepsilon} \quad \text { in } \mathbb{R}^{n}, \\
u>0 \text { in } \mathbb{R}^{n}, \\
u(y) \longrightarrow 0 \text { as }|y| \longrightarrow \infty,
\end{gathered}
$$

where $n \geq 3$ and $Q: \mathbb{R}^{n} \rightarrow \mathbb{R}$ is a positive continuous function. The aim is to construct high-energy solutions concentrating on a strict local minimum point $y_{0}$ of $Q(y)$.

In order to have the corresponding variational functional well defined in $\mathscr{D} 1,2\left(\mathbb{R}^{n}\right)$, the nonlinear term $u^{(n+2) /(n-2)+\varepsilon}$ in [16] is replaced by a nonhomogeneous term $f_{\varepsilon}(y, u)$, where the function $f_{\varepsilon}$ is defined as follows. Let $\gamma_{\varepsilon}: \mathbb{R} \rightarrow \mathbb{R}$ be a $C^{1}$ function such that

$$
\begin{aligned}
\gamma_{\varepsilon}(t) \geq 0 & \forall t \geq 0, \quad \gamma_{\varepsilon}(t)=0 \quad \forall t \geq 2, \\
\gamma_{\varepsilon}(t)=t^{(n+2) /(n-2)+\varepsilon} & \forall t \in[0,1], \quad \gamma_{\varepsilon}(t)=-\gamma_{\mathcal{\varepsilon}}(-t) \quad \forall t \in \mathbb{R},
\end{aligned}
$$

and set

$$
\bar{\gamma}_{\varepsilon}(y, t)=\frac{1}{|y|^{n+2+(n-2) \varepsilon}} \gamma_{\mathcal{\varepsilon}}\left(|y|^{n-2} t\right) \quad \forall y \in \mathbb{R}^{n}, \forall t \in \mathbb{R} .
$$

For a constant $K>0$ to be chosen later, let $h_{\varepsilon}: \mathbb{R} \rightarrow \mathbb{R}$ be the $C^{1}$ function defined by

$$
h_{\varepsilon}(t)= \begin{cases}t^{(n+2) /(n-2)+\varepsilon} & \text { if } 0 \leq t \leq \varepsilon^{-K n} \\ a_{\varepsilon} t^{(n+2) /(n-2)}+b_{\varepsilon} & \text { if } t \geq \varepsilon^{-K n} \\ -h_{\varepsilon}(-t) & \text { if } t \leq 0,\end{cases}
$$

where

$$
a_{\varepsilon}=\left(1+\frac{\varepsilon(n-2)}{n+2}\right) \varepsilon^{-K n \varepsilon}, \quad b_{\varepsilon}=\frac{\varepsilon(2-n)}{n+2} \varepsilon^{-K n((n+2) /(n-2)+\varepsilon)} .
$$


Then the modified nonlinearity $f_{\varepsilon}(y, t)$ is defined by

$$
f_{\mathcal{\varepsilon}}(y, t)=1_{B_{R}(0)} h_{\mathcal{\varepsilon}}(t)+1_{\mathbb{R}^{n} \backslash B_{R}(0)} \bar{\gamma}_{\mathcal{\varepsilon}}(y, t) \quad \forall y \in \mathbb{R}^{n}, \forall t \in \mathbb{R},
$$

where, for any set $A, 1_{A}$ denotes the characteristic function of $A$, and the radius $R$ is chosen large enough so that $B_{R}(0)$ contains $y_{0}$ (the strict local minimum point of $Q(y)$ ).

Notice that, for all $\varepsilon>0$, the variational functional related to the problem

$$
\begin{gathered}
-\Delta u=Q(y) f_{\varepsilon}(y, u) \quad \text { in } \mathbb{R}^{n}, \\
u>0 \quad \text { in } \mathbb{R}^{n}, \quad u \in \mathscr{D}^{1,2}\left(\mathbb{R}^{n}\right),
\end{gathered}
$$

is well defined and differentiable in $\mathscr{D}^{1,2}\left(\mathbb{R}^{n}\right)$.

Under suitable assumptions on $Q(y)$, in [16], it is obtained a $k$-peak solution for problem (2.28) by using a finite-dimensional reduction method. Then it is shown that, if in the definition of the function $h_{\varepsilon}$ the constant $K$ is chosen large enough, this solution also solves the original problem (2.22) when $\varepsilon$ is small enough.

We remark that the approximating problem (2.28) has critical growth for all $\varepsilon>0$; moreover, a Harnack inequality (see [4]) is used in [16] to obtain uniform estimates which guarantee that the solution obtained for problem (2.28) is actually a solution for problem (2.22) too, when $\varepsilon$ is small enough.

\section{Acknowledgments}

The authors are grateful to Professor Dancer for pointing out the article of Shusen Yan (after the submission of the first version of this paper). This work was supported by the Italian MIUR, National Research Project "Metodi variazionali e topologici nello studio di fenomeni non lineari."

\section{References}

[1] A. Bahri, Critical Points at Infinity in Some Variational Problems, Pitman Research Notes in Mathematics Series, vol. 182, Longman Scientific \& Technical, New York, 1989.

[2] A. Bahri and J.-M. Coron, On a nonlinear elliptic equation involving the critical Sobolev exponent: the effect of the topology of the domain, Comm. Pure Appl. Math. 41 (1988), no. 3, 253-294.

[3] A. Bahri, Y. Li, and O. Rey, On a variational problem with lack of compactness: the topological effect of the critical points at infinity, Calc. Var. Partial Differential Equations 3 (1995), no. 1, 67-93.

[4] H. Brézis and T. Kato, Remarks on the Schrödinger operator with singular complex potentials, J. Math. Pures Appl. (9) 58 (1979), no. 2, 137-151.

[5] H. Brézis and L. A. Peletier, Asymptotics for elliptic equations involving critical growth, Partial Differential Equations and the Calculus of Variations, Vol. I, Progr. Nonlinear Differential Equations Appl., vol. 1, Birkhäuser Boston, Massachusetts, 1989, pp. 149-192.

[6] M. Del Pino, P. Felmer, and M. Musso, Multi-peak solutions for super-critical elliptic problems in domains with small holes, J. Differential Equations 182 (2002), no. 2, 511-540.

[7] Z. Han, Asymptotic approach to singular solutions for nonlinear elliptic equations involving critical Sobolev exponent, Ann. Inst. H. Poincaré Anal. Non Linéaire 8 (1991), no. 2, 159-174.

[8] R. Molle and D. Passaseo, Positive solutions of slightly supercritical elliptic equations in symmetric domains, to appear in Ann. Inst. H. Poincaré Anal. Non Linéaire. 

, Positive solutions for slightly super-critical elliptic equations in contractible domains, C. R. Acad. Sci. Paris Sér. I Math. 335 (2002), no. 5, 459-462.

[10] On the existence of positive solutions of slightly supercritical elliptic equations, Adv. Nonlinear Stud. 3 (2003), no. 3, 301-326.

[11] O. Rey, A multiplicity result for a variational problem with lack of compactness, Nonlinear Anal. 13 (1989), no. 10, 1241-1249.

[12] Sur un problème variationnel non compact: l'effet de petits trous dans le domaine [On a variational problem with lack of compactness: the effect of small holes in the domain], C. R. Acad. Sci. Paris Sér. I Math. 308 (1989), no. 12, 349-352 (French).

[13] - The role of the Green's function in a nonlinear elliptic equation involving the critical Sobolev exponent, J. Funct. Anal. 89 (1990), no. 1, 1-52.

[14] Blow-up points of solutions to elliptic equations with limiting nonlinearity, Differential Integral Equations 4 (1991), no. 6, 1155-1167.

[15] - The topological impact of critical points at infinity in a variational problem with lack of compactness: the dimension 3, Adv. Differential Equations 4 (1999), no. 4, 581-616.

[16] S. Yan, High-energy solutions for a nonlinear elliptic problem with slightly supercritical exponent, Nonlinear Anal. Ser. A: Theory Methods 38 (1999), no. 4, 527-546.

Riccardo Molle: Dipartimento di Matematica, Università di Roma “Tor Vergata," Via della Ricerca Scientifica 1, 00133 Roma, Italy

E-mail address: molle@mat.uniroma2.it

Donato Passaseo: Dipartimento di Matematica “Ennio De Giorgi,” Università di Lecce, P.O. Box 193, 73100 Lecce, Italy 


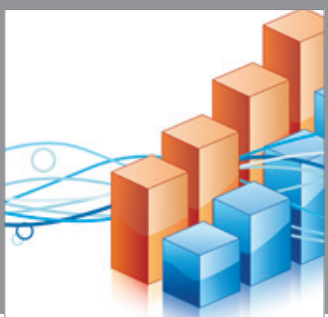

Advances in

Operations Research

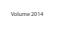

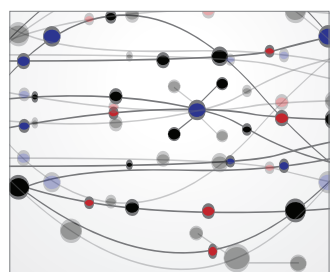

\section{The Scientific} World Journal
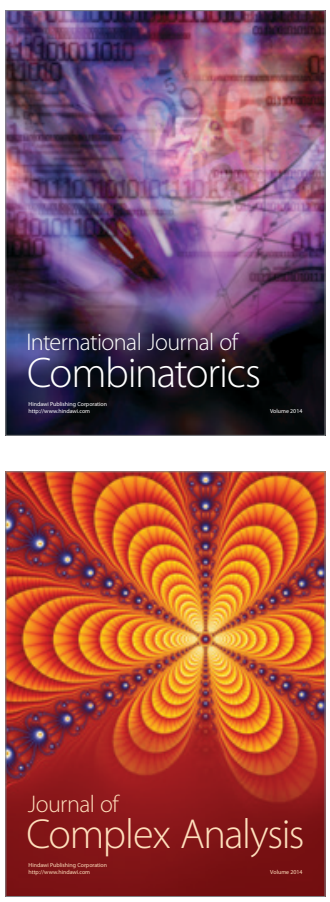

International Journal of

Mathematics and

Mathematical

Sciences
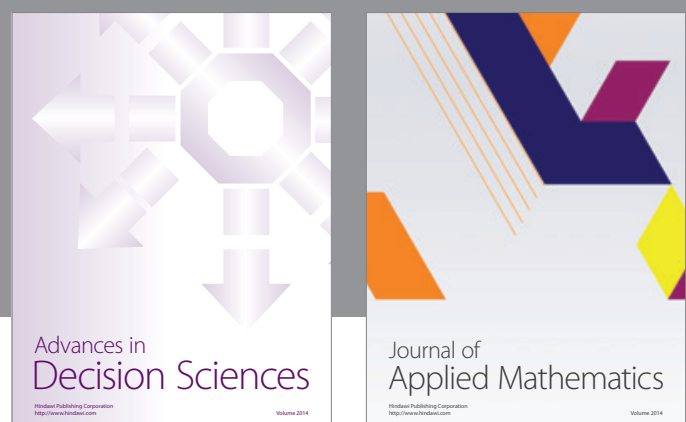

Journal of

Applied Mathematics
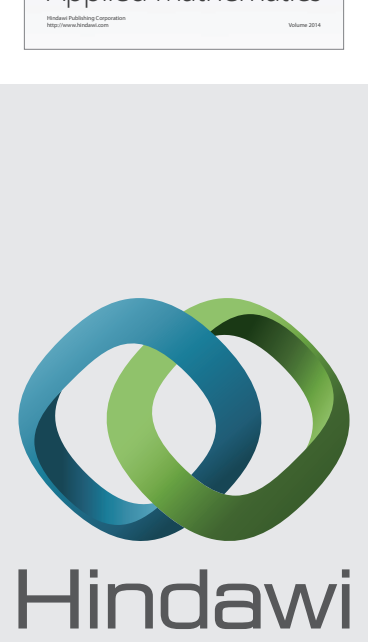

Submit your manuscripts at http://www.hindawi.com
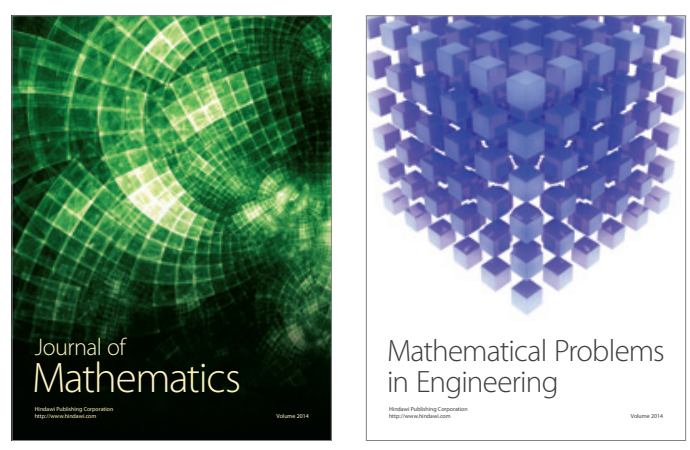

Mathematical Problems in Engineering
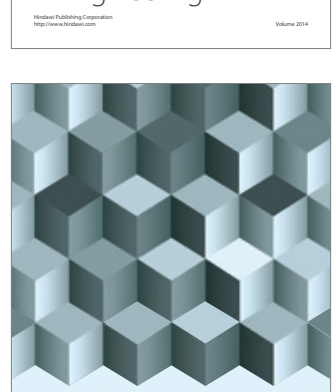

Journal of

Function Spaces
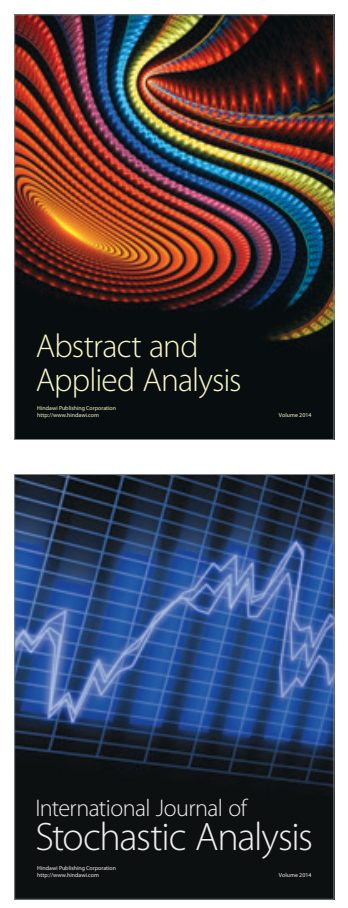

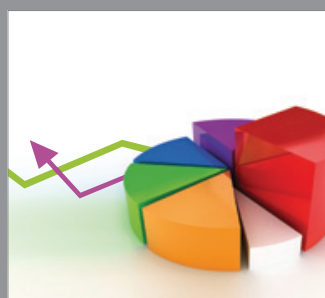

ournal of

Probability and Statistics

Promensencen
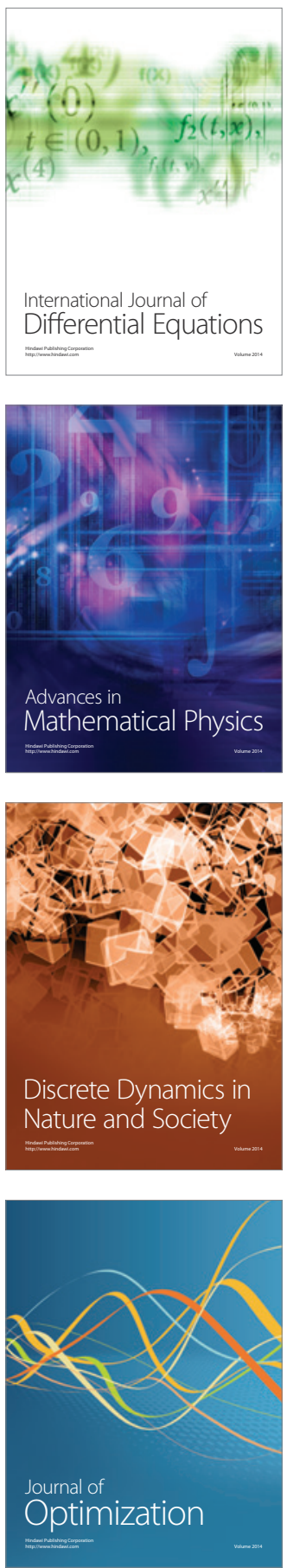